\title{
A Comprehensive Review on Routing in VANET
}

\author{
Sandeep Kumar Arora ${ }^{*}$ and Himanshu Monga \\ ${ }^{1,2}$ Discipline of Electronics and Communication Engineering, \\ Lovely Professional University, Jalandhar, Punjab, India-144411 \\ sandeep.16930@lpu.co.in, himanshumonga@gmail.com
}

\begin{abstract}
Vehicular Ad Hoc Networks (VANETs) have emerged as new technology with an aim of providing safety and security for the persons sitting in the vehicles. Consequently, wide variety of research contributions have been presented for VANET intrinsic characteristics. This paper provides comprehensive details of various types of routing protocol which is used to route the packets amongst various vehicles in high mobility scenarios. A detailed categorization of various routing techniques is provided in this paper with respect to the advantages, disadvantages and their various applications. The routing techniques have been compared to make the Intelligent Transportation System.
\end{abstract}

Keywords: VANET (Vehicular Adhoc Network), Routing protocols, Road Side Units(RSUs), On Board Units(OBUs), Mobility

\section{Introduction}

To share the information among the vehicles spontaneous network can be created among the vehicles which is known as VANET. There are two types of communication in a VANET network: (Vehicle to Vehicle) where vehicles can communicate directly and V2I (Vehicle to Infrastructure) where vehicles communicate directly with the existing infrastructure i.e., UMTS, GSM or WiMAX network. [1-2] Some standards which are already implemented in VANETs are WAVE and ETSI EN. It works without any coordination of infrastructure amongst vehicles. Transmission of messages involved routing schemes namely GeoUnicast, GeoBroadcast or Topologically scoped broadcast can be used. GeoUnicast messages are unicast to vehicles. In GeoBroadcast the intermediate nodes unicast the messages until it reaches the desired area where the message is broadcasted to reach to all vehicles. In topologically based type the message is broadcast to all vehicles in $\mathrm{n}$ hop neighborhood [3].

\subsection{Architecture and Components of VANETs}

The architecture of vehicular ad hoc network involves components like On Board Units (OBUs) and Road Side Units (RSUs) and Authentication Units (AUs). RSUs acts like a router while OBUs and AUs provides the services to RSUs. OBUs are installed in vehicles for the communication amongst RSUs. AUs are the sophisticated devices, which provides safety applications and communicate to RSU's by using OBUs. They provide Dedicated Short Range Communication (DSRC) using IEEE 802.11p. shown in Figure 1, The basic need of routing in VANET provides the information from one vehicle to another vehicle.

${ }^{*}$ Corresponding Author 


\subsection{Applications of Routing}

The applications of routing can be classified into three types viz. safety, transport efficiency and infotainment. Fast message transmission and collision avoidance lies under safety. Real time traffic monitoring is controlled by transport efficiency. Infotainment includes the information about nearby places.

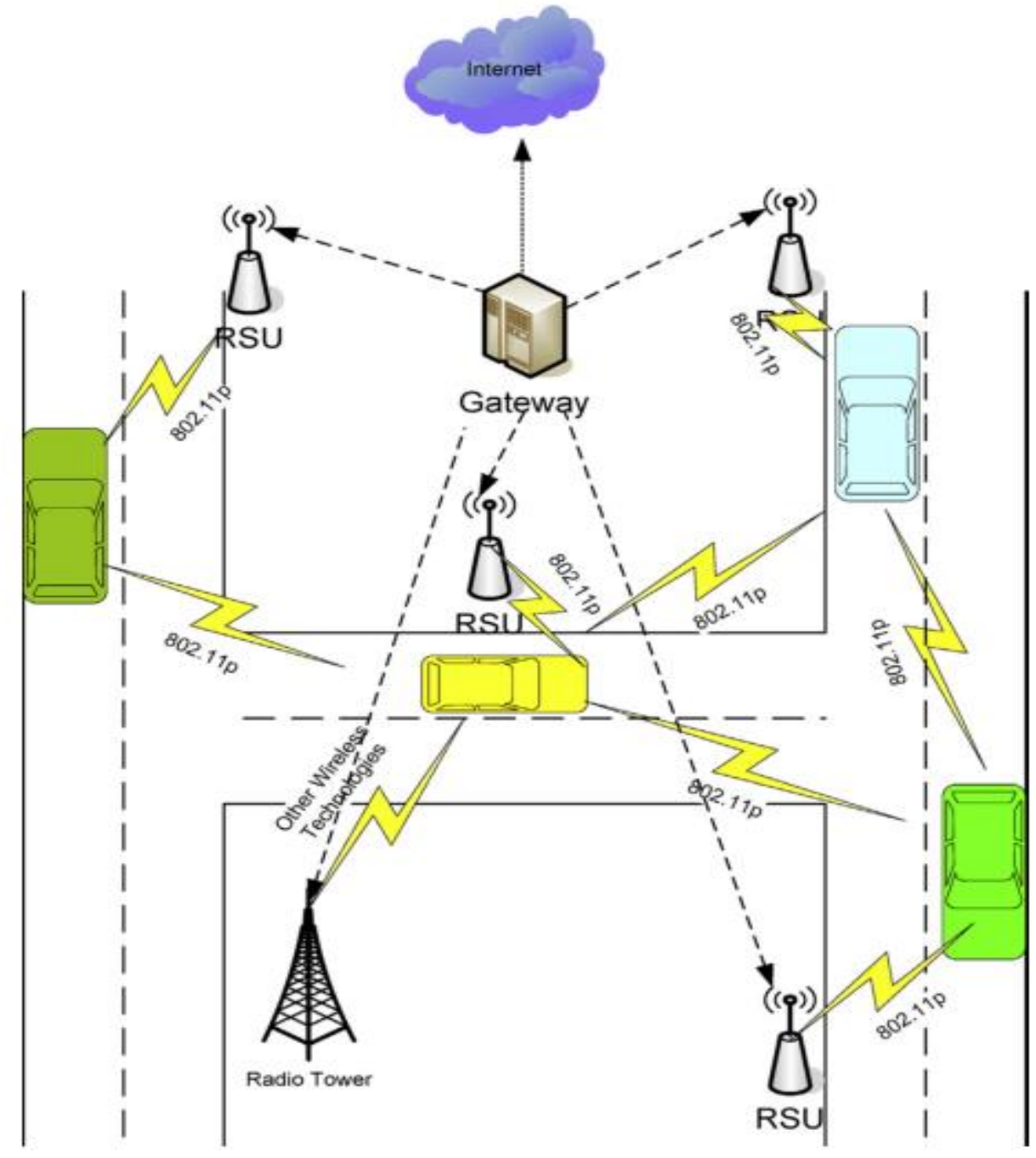

Figure 1. Architecture of VANET

\subsection{Constraint and Challenges}

Due to dynamic nature of VANET there are following constraints and challenges which ultimately effects the Quality of Services (QoS).

- Constant topological changes due to high mobility.

- Variation in the density and velocity of vehicles.

- Sparse distribution of vehicles in geographical regions which leads to poor connectivity.

- Selection of Cluster Head in cluster head communication.

- Detection of Intruders. 
So many researchers have proposed various techniques to overcome these problems which we will discuss in literature review [4].

\subsection{Taxonomy of Routing in VANETs}

Routing protocols can be categorized into various categories namely Topology, Geography, Hybrid, Clustering, Oppurtunistic and Data Fusion as shown in Figure 2. There are different approaches used for the efficient routing and to minimize the End to End delay and maximize the Quality of Services.

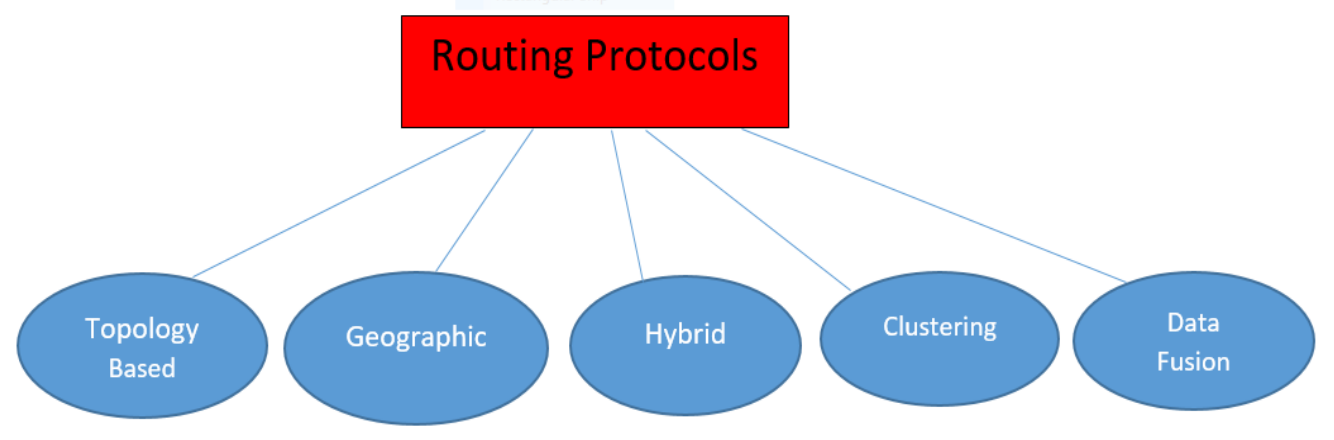

Figure 2. Taxonomy of Routing Protocols

\section{Topology Based Routing}

It defines that how the route is selected for sending the information from source to the destination. It can be classified as Proactive and Reactive protocols.

\subsection{Proactive Scheme}

In this scheme, every vehicle node maintains the routing table and finds the route with the help of routing table. Figure 3. depicts the classification of various topology based schemes. In this paper Prediction Based Routing(PBR) scheme is proposed in which the vehicles will create new routes as backup routes with the prediction algorithms which helps to minimize the failures [5].

In Destination Sequenced Distance Vector (DSDV) routing protocols, two routing tables are maintained at each node namely routing and setting table. Routing table has all the routing information like next hop, route metric and sequence number. Setting table has the information about the time of advertisement which is sent to the nodes [6].

OLSR is the routing protocol proposed in [7] which is designed for the optimization. It is following the proactive routing protocol strategy which is using the concept of flooding the control information using multipoint relays.

\subsection{Reactive Schemes}

This is basically known as route on demand which is consuming less bandwidth as compared to proactive type but it is slower than proactive due to non-availability of prior information. Following are the schemes under this category as shown in Figure 3,

Approach for Information Dissemination(AID) [8] in which the information from local nodes are gathered. This information includes the information about the neighbors and the distance between them. The proposed approach is evaluated on the basis of Saved Rebroadcasts(SRB), awareness and latency. AID is the better approach than other conventional techniques.

In this, researchers proposed a method through which the multimedia message can be transferred over the network passing by vehicles and integrates to the File Delivery Over Unidirectional Transport (FLUTE). First the data file is opened in binary mode. After the 
creation of File Delivery Table, there is a decomposition of encoding symbols into source symbols [9].

The authors presented [10] the enhanced Message Dissemination based on Roadmaps used to increase the percentage of informed vehicles and reduce the notification time. There are two algorithms proposed for sending and receiving purpose. In case of sending, if the vehicle is in warning mode then the message priority is set accordingly and in case of receiving if the consecutive gap between sender and receiver is more than the threshold value then message is rebroadcasted in this case.

SADV is proposed which is used for the transmission of messages using the optimal path. In this method the information is sent to the static node which will find the optimal path for sending that information to the destination. Road based Vehicular Traffic Reactive(RBVR-R) which is a combination of forwarding and route discovery [11].

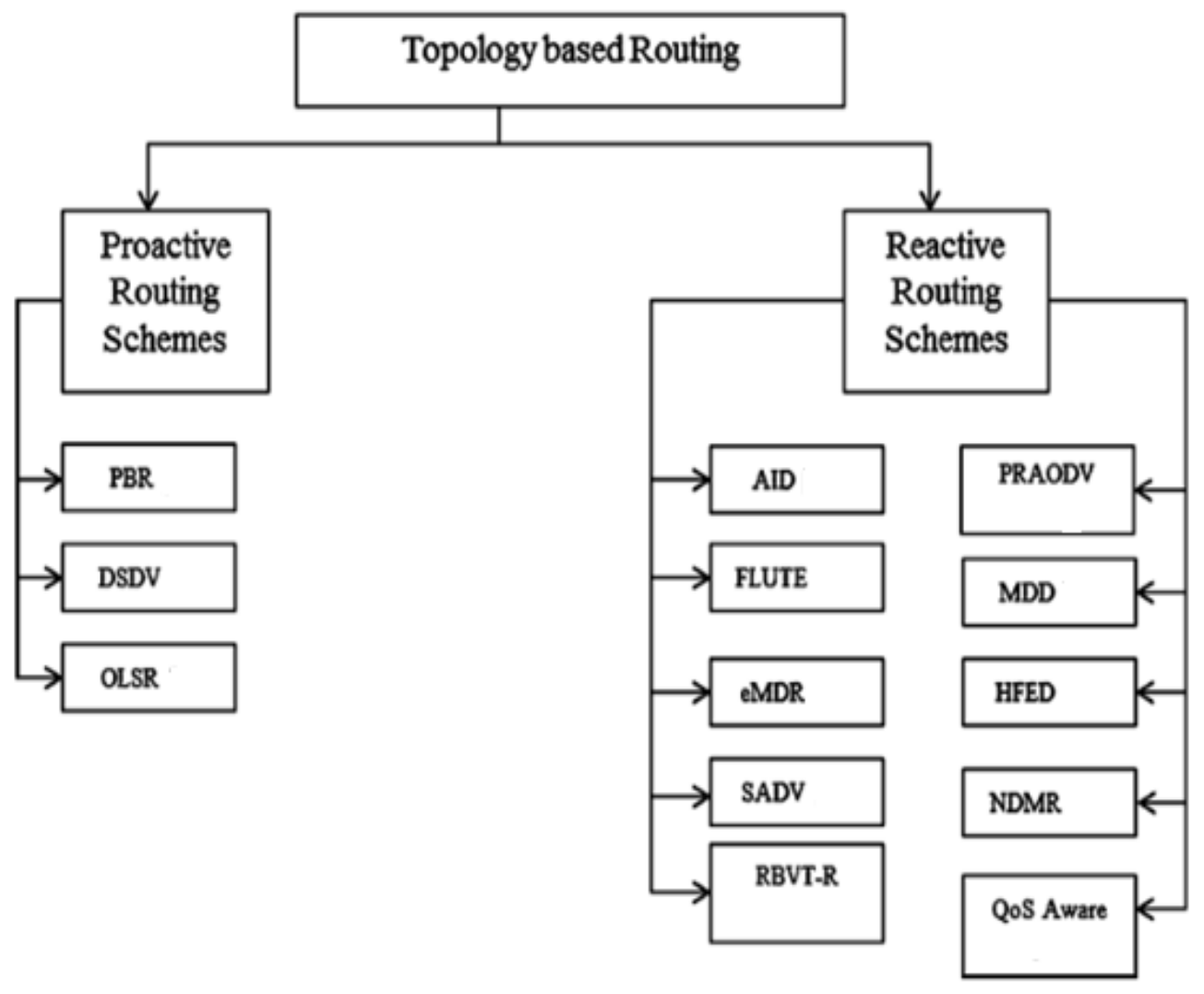

Figure 3. Classification of Topology based Scheme

To discover a path Source S sends the broadcast Route Request(RR). Once Destination receives $R R$, it replies with a Route Reply(RP) containing the connected path to it. In AODV alternate route is selected when there is a link failure but in PRAODV the alternative route is selected before the end of the route's link lifetime.

Bidirectional model has been proposed in which there are two affecting factors which affects the Message Delivery Delay(MDD), one is message delivery distance and other is traffic density. Hindering False Event Dissemination(HFED) is proposed to solve the problems like illusion, collusion, and Sybil attack. Sender must send the authenticated warning messages to the nodes and it is also known as proof of request. Node Disjoint Multipath Routing (NDMR) is used to examine the effect of mutual interference on the behavior of node disjoint paths. Simulation results shown in this there is an improvement for path selection in this algorithm. Multi-Protocol Label Switching (MPLS) can be used to find the suitable route which meets the requirements of traffic and Quality of Services (QoS). 


\section{Geographic Routing}

The geographic routing basically relies on the position of sender and receiver nodes. Now a days GPS is used to find the exact location of vehicles. If the location is known the packet can be sent without knowing the topology of the network. There are so many types of geographic routing but in this paper we have mentioned the most commonly used geographic routing protocols.

Anchor based Street and Traffic Aware Routing (A-STAR) proposed in [12] whose purpose is to support the routing in city environment. It finds the routes from the city bus routes using anchor path for higher connectivity so that more and more packets can be delivered. It is using the anchor path technique to find the new path from local maximum.

Greedy Perimeter Coordinator Routing(GPCR) using greedy approach [13] to find the best suited path. In this, the next hop is selected with the help of coordinator node which is helpful to route the packet to the destination. This technique is not useful where the traffic density is low.

Greedy Traffic Aware Routing (GYTAR) [14] is the intersection based routing approach used to find the route within the city. It uses digital map for the location and selects the junction on the basis on traffic density and curve metric distance. A score is given to every junction and the junction with the highest score will be elected as junction for forwarding the data packets. This approach is having the maximum connectivity which increases its Quality of Services(QoS).

Reliable Inter-Vehicle Routing (RIVER) approach utilizes the undirected graph approach representing the street graph. In this vertices are the curves and edges are street segments. Geographic Stateless VANET routing (GeoSVR) proposed to address the problem of local maximum in which relay node cannot find the neighbor close to the destination itself. Optimal path is selected from the source to destination having high traffic density between them. This protocol is using Dijkstra algorithm to find the shortest path with minimum weight as optimal path. In order to reduce the interference Free Space Path Loss(FSPL) is calculated as [15]:

$F S P L=20 \log d+20 \log f+118$

Where $\mathrm{d}$ is the communication distance in meters and $\mathrm{f}$ is the frequency in hertz.

$d=\frac{10^{\frac{F S P L}{\mathrm{NO}}-5.9}}{F}$

After proposing this, number of hops are increased and the PDR also increased but latency is $0.1 \mathrm{~s}$ only which is very less compared to other geographic techniques.

Vehicle Assisted Data Delivery (VADD) in which if there is no neighbor then packet has to be carried through road, then choose higher speed road. Dynamic path selection has been executed continuously. Figure 4 shows the scenario in VADD protocol in which vehicle $A$ has to send the data to vehicle $B$. Vehicle $A$ has two options either forward the packet to vehicle $\mathrm{B}$ which can be forwarded to D further which is moving towards destination or vehicle $\mathrm{A}$ can forward it to $\mathrm{C}$ which is moving towards the destination too There are two techniques Location First Probe(L-VADD) and Direction First Probe (DVADD). In Figure 4, according to L-VADD, the vehicle A will forward the packet to vehicle $\mathrm{B}$ which is the closest in North direction i.e., the direction of the destination. LVADD suffers from routing loops at the intersection area. As shown in Figure 7. DVADD protocol finds the vehicle, which is moving in the direction as its next packet carrier. Vehicle A will select vehicle $\mathrm{C}$ which is moving towards the direction of destination. [16]. 


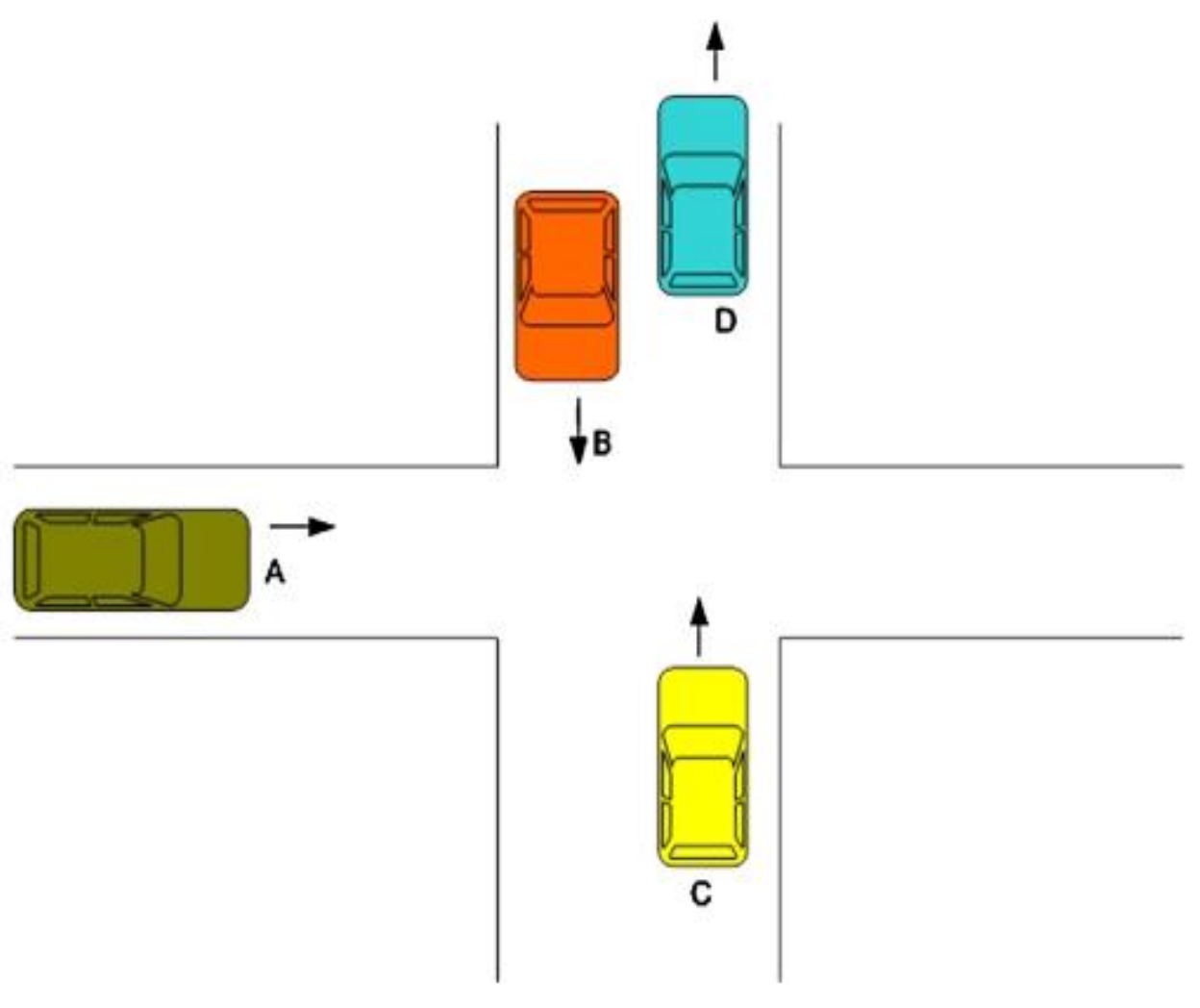

Figure 4. Section of the Next Vehicle to Forward the Packet

\section{Hybrid Protocol}

When Topology based and Geographic based protocols are combined they will generate Hybrid protocols. These are listed in Figure 5, below:

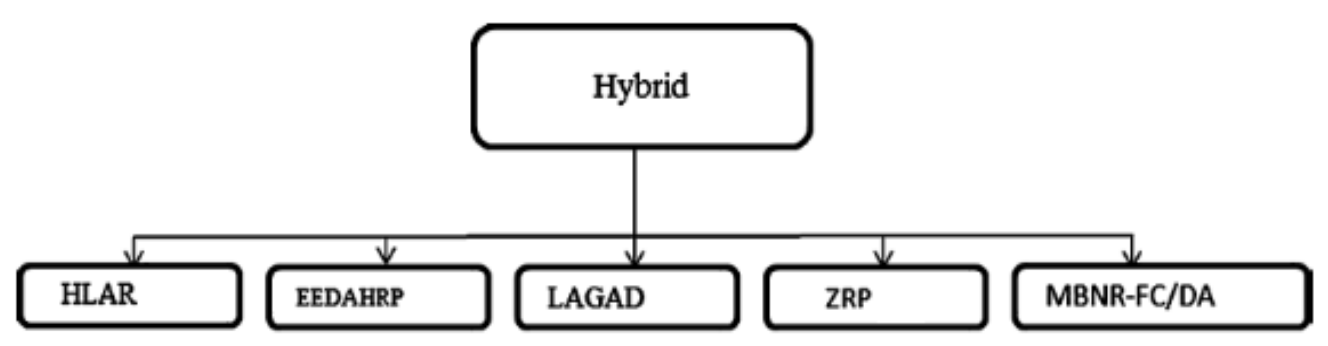

Figure 5. Classification of Hybrid Routing Protocols

Researchers proposed a scalable hybrid routing protocol for VANETs which combines the features of reactive routing and location based routing. Hybrid Location based Adhoc Routing protocol(HLAR) which combines AODV and Expected Transmission Count(ETX) parameters to find the best quality routes. Route discovery is done on the basis of on demand. It is having control packets similar to AODV i.e., RREQ, RREP and RERR. It has one more feature i.e., Route Repair(RREP) in which route is repaired locally when the link is broken. Routing over head in this is calculated as [17]:

$O_{i}=\frac{N_{i} S_{p}}{t}$

Where $\mathrm{Oi}$ is initiating overhead rate; $\mathrm{Ob}$ is beacon overhead rate; $\mathrm{Ni}$ is the total no. of packet transmission for initiating $\mathrm{m}$ communication pairs; $\mathrm{Sp}$ is control packet size. 
Location Aided Gateway Advertisement and Discovery(LAGAD) scheme is proposed in which there are following key features:

- It is built on the top of network layer.

- It uses channel diversity.

- It is based on the location aided adaptation of the advertisement zone of the gateway.

According to this scheme, it is assumed that every car is aware of its gateway and position is known for the vehicle. It is used to pass the information through the closest gateway and having very less overhead as compare to proactive, reactive and some other types of hybrid routing. But it suffers from the weakness in determining the efficient radius of gateway advertisement zone.

Zone Routing Protocol (ZRP) defines the zones containing the nodes that are most $\rho$ hops away. The nodes that are farther than $\rho$ hops away and don't lie in the zone are external nodes as shown in Figure 6 . The routing zone having $\rho=2$ centered at $S$, the nodes $\mathrm{A}$ to $\mathrm{H}$ are within 2 hop distance but are I, $\mathrm{J}$ and $\mathrm{K}$ are outside the zone [23].

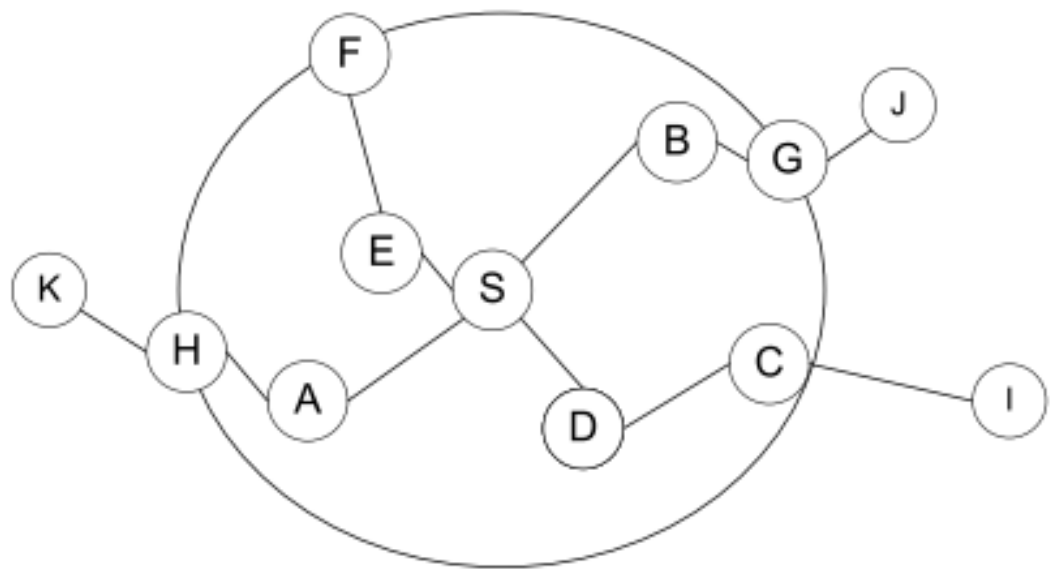

Figure 6. Routing Zone with $\rho=2$

IntrAzone Routing Protocol (IARP) maintains the up to date routing table within the zone. IntErzone Routing Protocol (IERP) is used for routing the data packets outside the zone. When the packets have to be sent globally, Broadcast Routing Protocol (BRP) is used. Nodes at the border of the zone are directly queried.

Mobile Backbone Network Routing with Flow Control and Distance Awareness (MBNR-FC/DA) which dynamically elects backbone capable nodes for routing the packets when the network fails. Mobile nodes make a network called mobile backbone(Bnet) in which nodes are dynamically elected to route the packets. This protocol uses center and global discovery process which reduces the control overhead of routing [18].

\section{Cluster Based Routing}

In this type of scheme there is role of Cluster Head $(\mathrm{CH})$ and member nodes which is managed by $\mathrm{CH}$. Cluster Head is useful for the communication with the another $\mathrm{CHs}$ and which again leads to communicate with member nodes. $\mathrm{CH}$ is selected with particular criteria like the node with high stability factor. In this section we presented some cluster based protocols.

LORA_CBR is proposed in which routing is performed which is based on greedy manner. Location Request (LREQ) are sent by the source nodes. $\mathrm{CHs}$ are useful to 
disseminates LREQ and Location Reply(LREP) packets. This protocol is similar to AODV but the only difference is only CHs will disseminate the information [19].

Fault Tolerant Location based Vehicular Service Delivery Protocol(FTLocVSDP) in which the main consideration is fault tolerance. Due to faulty components between the service provider and requester the packet drop is high. To overcome that drop this scheme is proposed. In this, the requester specifies the region of interest within the request and the protocol uses the discovery of location based services. After implementing this technique researcher found that there is an improvement of 50\% in communication link failure [20].

Cooperation Communication Aware Link Scheduling for Cognitive VANET [21] (CVANET) and have investigated and throughput maximization problem such as:

- The availability of spectrum.

- Cognitive Radio signal ratio constraint.

- Transmission mode selection and Link scheduling.

Adaptive Role Playing (ARP) strategy to enable the nodes in each hop to contend to perform the basic function. The authors also proposed the LEAPER, which enables the nodes in each hop to securely and efficiently follow ARP strategy [22].

\section{Data Fusion Based Routing}

Data fusion is the technique which reduces the data from the redundant nodes. Data fusion techniques has been categorized in Figure 7, given below:

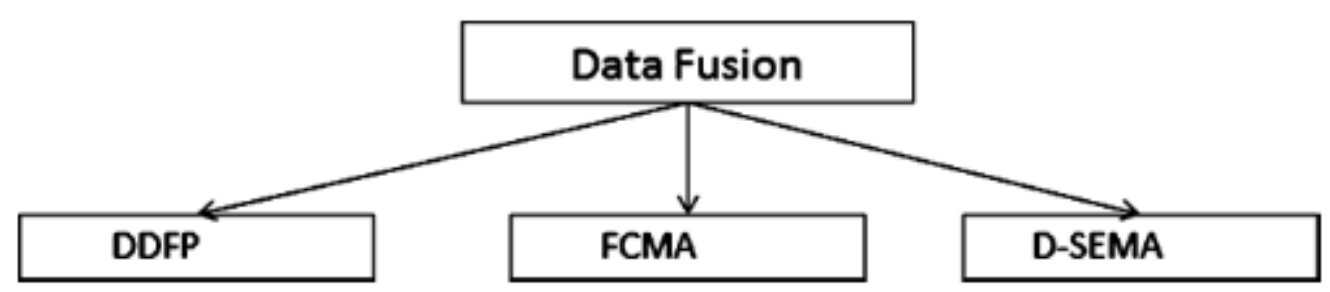

Figure 7. Classifications of Data Fusion Schemes

Driver in-the-loop Data Fusion Problem (DDFP) is proposed in which there are Data Elements (DEs) and Auxiliary part. All the messages must reach to the driver for whom it is generated. But the limitation is limited number of messages can be transmitted. So this problem is overcome by DDFP.

Multilevel fusion approached is proposed by using Fuzzy Clustering Message Aggregation (FCMA) in which local atomic messages are classified into different message clusters and abnormalities can be extracted. In this Basic Probability Assignment is calculated. This BPA value is and abnormal aggregation message. The basic advantage of FCMA is it can easily distinguish the different traffic conditions. Authors have proposed another algorithm i.e., D-S Evidence Message Aggression (D-SEMA) that removes the false abnormal feature information caused by the traffic lights.

\section{Conclusion}

Over the last few decades, VANETs have emerged as new powerful technology which can be used in so many applications like Rescue and surveillance operation, entertainment etc. But routing is the major part of VANET. In this paper, we analyzed the various routing schemes and also elaborated their advantages and disadvantages. We analyzed that which protocol performs better in high traffic density and producing lesser overhead. 
In future, we would implement one of the above routing schemes and compare its performance over the other schemes.

\section{References}

[1] H. Yoo and D. Kim, "Repetition-based cooperative broadcasting for vehicular ad-hoc networks", Comput. Commun., vol. 34, no. 9, (2011), pp. 1870-1882.

[2] H. Fubler, M. Mauve, H. Hartenstein, M. Kasemann and D. Vollmer, "Location-based routing for vehicular ad-hoc networks", Proceedings of the Mobile Computing and Communications Review, SIGMOBILE, New York, USA, September (2002).

[3] ETSI $\quad$ EN $302 \quad 636$, http://www.etsi.org/deliver/etsi_en/302600_302699/30263601/01.02.00_20/en_30263601v010200a.pdf, (2013).

[4] S. Sultan, M. Doori, A. Bayatti and H. Zedan, "A comprehensive survey on vehicular ad hoc networks", Journal of Networking and Computer Application, (2013).

[5] V. Namboodiri and L. Gao, "Prediction-based routing for vehicular ad hoc networks", IEEE Trans. Veh. Technol., vol. 56, no. 4, (2007), pp. 2332-2345.

[6] C. E. Perkins and P. Bhagwat, "Highly dynamic destination sequence distance-vector routing (DSDV) for mobile computers", Proceedings of Conference on Communications Architectures, Protocols and Applications (SIGCOMM '94), New York, USA, (1994) October.

[7] J. Toutouh, J. G. Nieto and E. Alba, "Intelligent OLSR routing protocol optimization for VANETs", IEEE Trans. Veh. Technol., vol. 61, no. 4, (2012), pp. 1884-1894.

[8] M. Bakhouya, J. Gaber and P. Lorenz, "An adaptive approach for information dissemination in vehicular ad hoc networks”, Journal of Netw. Comput. Appl., vol. 34, no. 6, (2011), pp. 1971-1978.

[9] C. T. Calafate, G. Fortino, J. Monteiro, J. C. Cano and P. Manzoni, "An efficient and robust content delivery solution for IEEE 802.11p vehicular environments", Journal of Netw. Comput. Appl., vol. 35, no. 2, (2012), pp. 753-762.

[10] M. Fogue, P. Garrido, F. J. Martinez, J. C. Cano, C. T. Calafate and P. Manzoni, "Evaluating the impact of a novel message dissemination scheme for vehicular networks using real maps", Transp. Res., Part C, Emerg. Technol., vol. 25, (2012), pp. 61-80.

[11] Y. Ding, C. Wang and L. Xiao, "A static-node assisted adaptive routing protocol in vehicular networks", Proceedings of Fourth ACM International Workshop on Vehicular Ad hoc Networks VANET'07, Montreal, Canada, (2007).

[12] C. Lochert, M. Mauve, H. Fler and H. Hartenstein, "Geographic routing in city scenarios", (poster), MobiCom 2004, ACM SIGMOBILE Mobile Computing and Communications Review (MC2R), vol. 9, no. 1 , (2005), pp. 69-72.

[13] M. Jerbi, S. M.Senouci, R. Meraihi and G. D. Yacine, "An improved vehicular Ad hoc routing protocol for city environments", Reviewed at the Direction of IEEE International Conference on Communications, (2007), pp. 3972-3979.

[14] J. Bernsen and D. Manivannan, "RIVER: A reliable inter-vehicular routing protocol for vehicular ad hoc networks", Comput. Netw., vol. 52, no. 17, (2012), pp. 3795-3807.

[15] J. Zhao and G. Cao, "VADD: Vehicle-assisted data delivery in vehicular ad hoc network", IEEE Trans. Veh. Technol., vol. 57, no. 3, (2008), pp. 1910-1922.

[16] M. A. Rabayah and R. Malaney, "A new scalable hybrid routing protocol for VANETs", IEEE Trans. Veh. Technol., vol. 61, no. 6, (2012), pp. 2625-2635.

[17] X. Huang and I. Rubin, "Mobile backbone network routing with flow control and distance awareness (MBNR-FC/DA)", Proceedings of IEEE Military Communications Conference, MILCOM 2005, Atlantic City, NJ, (2005)

[18] R. A. Santos, A. Edwards, R. Edwards and L. Seed, "Performance evaluation of routing protocols in vehicular ad hoc networks", International Journal of Ad Hoc Ubiq. Comput., vol. 1, no. 2, (2005), pp. 80-91.

[19] K. Abrougui, A. Boukerche and H. Ramadan, "Performance evaluation of an efficient fault tolerant service discovery protocol for vehicular networks", Journal of Netw. Comput. Appl., vol. 35, no. 5, (2012), pp. 1424-1435.

[20] M. Pan, P. Li and Y. Fang, "Cooperative communication aware link scheduling for cognitive vehicular networks", IEEE J. Sel. Areas Commun., vol. 30, no. 4, (2012), pp. 760-768.

[21] Z. Li and C. Chigan, "LEAPER: a lightweight reliable and faithful data packet relaying framework for VANETs", Ad Hoc Netw. vol. 9, no. 3, (2011), pp. 418-429.

[22] A. Dua, N. Kumar and S. Bawa, "A systematic review on routing protocols for VANET", Vehicular Communications, vol. 1, no. 1, (2014), pp. 33-52. 


\section{Authors}

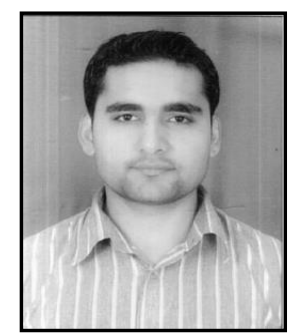

Sandeep Kumar Arora, is currently pursuing Ph. D. in Electronics \& Electrical Engineering with Spl. in Design of Secure Initiation Protocol in VANET. He is working as an Asst. Prof. in Lovely Professional University since 2011. His research interest includes Wireless Sensor Networks, Computer Networks, Adhoc Networks Communications and Cryptography. He is a member of IEEE and also the author of more than one dozen research papers indexed in Scopus.

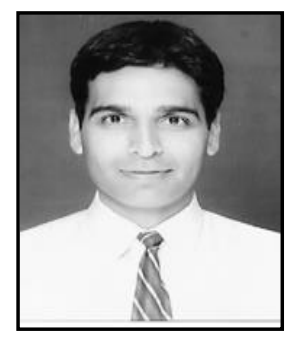

Himanshu Monga has obtained Ph.D. from Thapar Institute of Engineering and Technology, Department of ECE, Patiala, India in the field of Optical and wireless networks. He is presently working as a Professor and Associate Dean in Lovely Professional University, Punjab, India in the department of R\&D. He is member of IEEE and ISTE. He is an author of more than 65 papers. 